\title{
Comment on "APOBEC3B interaction with PRC2 modulates microenvironment to promote HCC progression"
}

\author{
Beiying Dai \\ Center for New Drug Safety Evaluation and Research, State Key Laboratory of Natural Medicines, China Pharmaceutical \\ University, Nanjing 211198, Jiangsu, China.
}

Correspondence to: Prof. Beiying Dai, Center for New Drug Safety Evaluation and Research, State Key Laboratory of Natural Medicines, China Pharmaceutical University, 639 Longmian Avenue, Nanjing 211198, Jiangsu, China.

E-mail: 1620184503@cpu.edu.cn

\begin{abstract}
How to cite this article: Dai Beiying. Comment on "APOBEC3B interaction with PRC2 modulates microenvironment to promote HCC progression". Hepatoma Res 2019;5:33. http://dx.doi.org/10.20517/2394-5079.2019.26
\end{abstract}

Received: 11 Jul 2019 First Decision: 11 Jul 2019 Revised: 11 Jul 2019 Accepted: 12 Jul 2019 Published: 4 Sep 2019

Science Editor: Guang-Wen Cao Copy Editor: Cai-Hong Wang Production Editor: Jing Yu

Accounting for $75 \%-85 \%$ of all primary liver cancer cases, hepatocellular carcinoma (HCC) is nowadays one leading cause of cancer-related mortality worldwide ${ }^{[1]}$. More than half of HCC patients are diagnosed at the advanced stage, for which limited treatment options are available and no curative ones exist so far, leading to poor prognosis ${ }^{[2]}$. The main risk factors for HCC, such as infection with HBV and HCV, excessive alcohol consumption, obesity, and diabetes, all contribute to chronic liver inflammation, which leads to the formation of an altered liver microenvironment. In turn, an altered liver microenvironment can reciprocally reprogram the immune cells and hepatocytes involved in inflammation, together setting the stage for progression to cirrhosis and eventually to $\mathrm{HCC}^{[2-4]}$.

It has been demonstrated that tumor-associated macrophages (TAMs) and myeloid-derived suppressor cells (MDSCs) are the most abundant immune cell populations infiltrated in the tumor microenvironment of HCC. As pivotal players in cancer-related inflammation, TAMs and MDSCs promote hepatocarcinogenesis by stimulating angiogenesis and inducing immunosuppression and correlate with inferior prognosis ${ }^{[5-7]}$. Thus, it is of crucial importance to gain an in-depth look at the interplay between hepatocytes and immune cells, especially TAMs and MDSCs, during the development of HCC.

Recently Wang et al ${ }^{[8]}$ presented a remarkable study unraveling the functional significance of hepatocyteintrinsic apolipoprotein B mRNA editing enzyme catalytic polypeptide-like 3B [APOBEC3B (A3B)] in

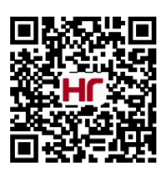


promoting HCC progression by recruiting TAMs and MDSCs to the tumor microenvironment, thus inhibiting $\mathrm{CD}^{+} \mathrm{T}$ cell function and further facilitating immune escape.

In this issue, the authors first demonstrated elevated abundance of $\mathrm{A} 3 \mathrm{~B}$ in HCC patients due to overactivation of the non-classical NF-кB pathway and direct transcriptional regulation by RelB. Taking advantage of both immunocompetent and immune-deficient mouse HCC models, they revealed that A3B activated $\mathrm{HCC}$ initiation through modulation of the immune system as $\mathrm{A} 3 \mathrm{~B}$ exerted its carcinogenic function only in mice with complete immune system, which was accompanied by increased secretion of CCL2, IL-34 and BMP7 and the subsequent accumulation of TAMs, MDSCs and Programmed cell death $1(\mathrm{PD} 1)^{+} \mathrm{CD} 8 \mathrm{~T}$ cells.

After establishing the impact of hepatocyte-intrinsic A3B on immunological environment in HCC development, Wang and his colleagues performed a series of analyses to investigate the molecular basis of this phenomenon. They found out that A3B inhibited PRC2 activity through both interference of its binding affinity and attenuating its enzymatic activity, while PRC2 has been reported to be indispensable in the methylation of $\mathrm{H}_{3} \mathrm{~K} 27$ and regulate chemokine expression ${ }^{[9,10]}$. Bioinformatic analyses showed a highly overlapping cohort of target genes, whose expression levels altered in inverse correlation upon exogenous $\mathrm{A} 3 \mathrm{~B}$ expression and $\mathrm{H}_{3} \mathrm{~K} 27 \mathrm{me} 3$. Experiments further demonstrated $\mathrm{H} 3 \mathrm{~K} 27 \mathrm{me} 3$ sites at the promoter regions of CCL2, IL-34 and BMP7. Taken together, upregulated A3B suppressed occupancy of H3K27me3 on the promoter of chemokines CCL2, IL-34 and BMP7 by inhibiting PRC2 activity.

In last decade, immunotherapy dramatically revolutionized the therapeutic landscape in oncology and was announced as Breakthroughs of the Year by Science in 2013. However, the progress of introducing either chimeric antigen receptor (CAR)-modified T cells or checkpoint inhibitors into HCC therapy is rather slow. In 2017, Nivolumab was approved as the only anti-PD-1/L1 antibody for the treatment of HCC patients ${ }^{[11]}$. However, the response rate reached only about $20 \%{ }^{[12]}$. One major cause of such low effectiveness lies in the immunosuppressive tumor microenvironment and immune escape. In addition to immunotherapy, epigenetic therapy has drawn much attention in recent years as well. However, outcome of pre-clinical and clinical trials of epigenetic drugs in HCC was rather disappointing, indicating other molecular mechanisms involved in epigenetic modulation ${ }^{[13]}$. This work by Wang and his colleagues discovered a crucial role of $\mathrm{A} 3 \mathrm{~B}$ in promoting HCC initiation by modulating immunological microenvironment via inhibition of $\mathrm{H}_{3} \mathrm{~K} 27$ methylation, revealing $\mathrm{A} 3 \mathrm{~B}$ as a novel therapeutic target in immunotherapy of HCC, explaining partially the current failure of epigenetic drugs, and demonstrating the significance of combined therapy targeting both innate and acquired immune systems in future HCC treatment.

\section{DECLARATIONS}

\section{Authors' contributions}

The author contributed solely to the article.

\section{Availability of data and materials}

Not applicable.

\section{Financial support and sponsorship}

None.

\section{Conflicts of interest}

The author declared that there are no conflicts of interest. 


\section{Ethical approval and consent to participate}

Not applicable.

\section{Consent for publication}

Not applicable.

\section{Copyright}

(C) The Author(s) 2019.

\section{REFERENCES}

1. Bray F, Ferlay J, Soerjomataram I, Siegel RL, Torre LA, et al. Global cancer statistics 2018: GLOBOCAN estimates of incidence and mortality worldwide for 36 cancers in 185 countries. CA Cancer J Clin 2018;68:394-424.

2. Llovet JM, Montal R, Sia D, Finn RS. Molecular therapies and precision medicine for hepatocellular carcinoma. Nat Rev Clin Oncol 2018;15:599-616.

3. Prieto J, Melero I, Sangro B. Immunological landscape and immunotherapy of hepatocellular carcinoma. Nat Rev Gastroenterol Hepatol 2015;12:681-700.

4. Llovet JM, Zucman-Rossi J, Pikarsky E, Sangro B, Schwartz M, et al. Hepatocellular carcinoma. Nat Rev Dis Primers 2016;2:16018.

5. Wan S, Kuo N, Kryczek I, Zou W, Welling TH. Myeloid cells in hepatocellular carcinoma. Hepatology 2015;62:1304-12.

6. Lu LC, Chang CJ, Hsu CH. Targeting myeloid-derived suppressor cells in the treatment of hepatocellular carcinoma: current state and future perspectives. J Hepatocell Carcinoma 2019;6:71-84.

7. Degroote H, Van Dierendonck A, Geerts A, Van Vlierberghe H, Devisscher L. Preclinical and Clinical Therapeutic Strategies Affecting Tumor-Associated Macrophages in Hepatocellular Carcinoma. J Immunol Res 2018;2018:7819520.

8. Wang D, Li X, Li J, Lu Y, Zhao S, et al. APOBEC3B interaction with PRC2 modulates microenvironment to promote HCC progression. Gut 2019; doi: 10.1136/gutjnl-2018-317601.

9. Cao R, Zhang Y. SUZ12 is required for both the histone methyltransferase activity and the silencing function of the EED-EZH2 complex. Mol Cell 2004;15:57-67.

10. Nagarsheth N, Wicha MS, Zou W. Chemokines in the cancer microenvironment and their relevance in cancer immunotherapy. Nat Rev Immunol 2017;17:559-72.

11. Nivolumab Approved for Liver Cancer. Cancer Discov 2017;7:OF3.

12. Chen S, Cao Q, Wen W, Wang H. Targeted therapy for hepatocellular carcinoma: Challenges and opportunities. Cancer Lett 2019;460:1-9.

13. Toh TB, Lim JJ, Chow EK. Epigenetics of hepatocellular carcinoma. Clin Transl Med 2019;8:13. 